\title{
Multi-scale Correlation Functions in Strong Turbulence
}

\author{
Jahanshah Davoudi ${ }^{a, c, d}$ and M. Reza Rahimi Tabar ${ }^{b, c, d}$ \\ ${ }^{a}$ Dept. of Physics, Sharif University of Technology, P.O.Box 11365-9161, Tehran, Iran. \\ ${ }^{b}$ CNRS UMR 6529, Observatoire de la Côte d'Azur, BP 4229, 06304 Nice Cedex 4, France, \\ ${ }^{c}$ Dept. of Physics, Iran University of Science and Technology, Narmak, Tehran 16844, Iran. \\ ${ }^{d}$ Institue for Studies in Theoretical Physics and Mathematics Tehran P.O.Box: 19395-5746, Iran,
}

\begin{abstract}
Under the framework of V. Yakhot [Phys.Rev.E, 57, 1737 (1998)] modelling of intermittent structure functions in fully developed turbulence and based on the experimentally supported Markovian nature of turbulence cascades [R.Friedrich and J.Peinke, Phys.Rev.Lett, 78, 863 (1997)], we calculate the multiscaling correlation functions. Fusion rules [V.S.L'vov and I. Procaccia, Phys.Rev.Lett. 76, 2898 (1996)] which are experimentally tested [R. Benzi, L. Biferale, and F. Toschi, Phys.Rev.Lett. 80, 3244 (1998)] to be compatible with almost uncorrelated multiplicative process can analyticaly be supported by direct calculations.

PACS numbers, 47.27.Ak, 47.27.Gs, 47.27.Jv and 47.40.Ki.
\end{abstract}

One of the most important issues in stationary turbulence is the intermittent behaviour of the velocity fluctuations in the inertial range. Understanding the statistical properties of intermittency is one of the most challenging open problems in three dimensional fully developed turbulence. The structures that arise in a random flow of stationary turbulence resembel themselves as high peaks at random places and random times. The intervals between them are characterised by a low intensity and a large size. Rare peaks are the hallmarks of PDF's nonguassian tails. These strongly non-Gausssian activities are statistically scale-invariant processes responsible for energy transfer. Intermittency in the inertial range is usually analysed by means of the statistical properties of velocity differences, $\delta_{r} u(x)=u(x+r)-u(x)$ 11. The overwhelming experimental and theoretical works have been brought forward for characterisation of structure functions; i.e. $S_{p}=\left\langle\left(\delta_{r} u(x)\right)^{p}\right\rangle$. A wide agreement exists on the fact that $S_{p}(r)$ exhibits a scaling behaviour in the limit of high Reynolds number, that is $S_{p}(r) \sim\left(\frac{r}{L}\right)^{\zeta_{p}}$ for $L>>r>>\eta_{k}$, where $L$ is the scale of energy injection, $\eta_{k}=\left(\frac{\nu^{3}}{\epsilon}\right)^{1 / 4}$ is the dissipative scale, $\epsilon$ is the mean energy dissipation range and $\nu$ is the kinematic viscosity. Rare peaks in the random flows are signaled with a nonlinear form of $\zeta(p)$. In other words the velocity increments are multifractal and $\zeta(p)$ 's do not follow the celebrating K41 theory, $\zeta(p)=p / 3$. Recently [2 4 it has been proposed that it would be more natural to look at single time correlations among velocity increment fluctuations at different scales:

$$
\mathcal{F}_{n}\left(x \mid r_{1}, r_{2}, \ldots, r_{n}\right)=\left\langle\delta_{r_{1}} u(x) \delta_{r_{2}} u(x) \ldots \delta_{r_{n}} u(x)\right\rangle
$$

where all the scales $r_{i}$ are lying in the inertial range,i.e. $\eta<<r_{i}<<L$ For simplicity we are confining the discussion to longitudinal velocity increments. Fusion rules [2 4 ] that describe the asymptotic properties of n-point correlation functions when some of the coordinates tend toward one other are derived from two fundamental assumptions which are of paramount importance for the description of non-perturbative aspects of the analytic theory of stationary turbulence. The fusion rules are tested experimentaly and a good agreement between experiment and theory have been observed [5]. If $p<n$ pairs of coordinates of velocity differences merge, with the typical separations $r_{i} \sim r$ for $i \leq p$ and the remaining separation at the order of $R$ such that $r<<R<<L$ , the fused multiscale correlation is defined as

$$
\begin{aligned}
\mathcal{F}_{p+q}(r, R) & \equiv\left\langle(u(x+r)-u(x))^{p}(u(x+R)-u(x))^{q}\right\rangle \\
& \equiv\left\langle\left(\delta_{r} u(x)\right)^{p}\left(\delta_{R} u(x)\right)^{q}\right\rangle
\end{aligned}
$$

it has been deduced that

$$
\mathcal{F}_{p+q}(r, R) \sim S_{p}(r) S_{p+q}(R) / S_{p}(R)
$$

On the other hand recently multiscale correlation functions in high Reynolds number experimental turbulence, numerical simulations and synthetic signal was investigated by Benzi and coworkers [6] and it has been found that whenever the simple scaling ansatz based on the uncorrelated multiplicative processes [6] is not prevented by symmetry arguments, the multiscale correlations are in good agreement with the fusion rules prediction even if strong corrections due to subleading terms are seen for small-scale seperation $r / R \sim O(1)$. All the findings has led to the following conclusion that multiscale correlation functions measured in turbulence are fully consistent with a multiplicative almost uncorrelated random process for evolution of velocity increments in scale. Although successful interpretation of the fusion rules can be realised by considering a multiplicative random process for the evolution of velocity increments in length scale, but it is at most a phenomenological modelling and it is not based on a first principle calculations. Other front of experimental investigations over the bahaviour of conditional probability densities of velocity increments in scale have shown that Markovian nature of velocity increments in terms of length scale and in the inertial range would support the experimental data 7 . In fact the necessary 
condition of "Markovianity" for velocity increments have been tested experimentaly from which phenomenological senarios for modelling the intermittency have been developed [7]. Afterwards the aforementioned ideas were supported by invoking to the theoretical ideas inspired by Polyakov [8] and Yakhot [9] based on OPE and general invariances of the Navier-Stokes equation [9]. In this paper we show that the interpretation given by Benzi and coworkers [6] dictating an uncorrelated multiplicative process for velocity increments in the inertial range is not just a mere phenomenological interpretation and can be supported by Yakhot modelling. Even we will candidate the underlying dynamical process in scale which incorporates the fusion rules of multiscale correlation functions. The calculations are consistent with the picture of almost uncorrelated random multiplicative process at least in the Fokker-Planck description of turbulence cascades. Furthermore we are able to connect the fusion rules to the Markovian nature by a simple operator formalism even by perserving all the terms in the KramersMoyal's evolution operator of velocity increments.

Let us start with the Navier-Stokes equation:

$$
\mathbf{v}_{t}+(\mathbf{v} \cdot \nabla) \mathbf{v}=\nu \nabla^{2} \mathbf{v}-\frac{\nabla p}{\rho}+\mathbf{f}(\mathbf{x}, t), \quad \nabla \cdot \mathbf{v}=\mathbf{0}
$$

for the Eulerian velocity $\mathbf{v}(\mathbf{x}, t)$ and the pressure $p$ with viscosity $\nu$, in $\mathrm{N}$-dimensions. The force $\mathbf{f}(\mathbf{x}, t)$ is the external stirring force, which injects energy into the system on a length scale $L$. More specifically one can take, for instance a Gaussian distributed random force, which is identified by its two moments:

$$
\left\langle f_{\mu}(\mathbf{x}, t) f_{\nu}\left(\mathbf{x}^{\prime}, t^{\prime}\right)\right\rangle=k(0) \delta\left(t-t^{\prime}\right) k_{\mu \nu}\left(\mathbf{x}-\mathbf{x}^{\prime}\right)
$$

and $\left\langle f_{\mu}(\mathbf{x}, t)\right\rangle=0$, where $\mu, \nu=x_{1}, x_{2}, \cdots, x_{N}$. The correlation function $k_{\mu \nu}(r)$ is normalized to unity at the origin and decays rapidly enough where $r$ becomes larger or equal to integral scale $L$, that is $k_{\mu \nu}\left(r_{i j}\right)=k(0)[1-$ $\left.\frac{r_{i j}^{2}}{2 L^{2}} \delta_{\mu, \nu}-\frac{\left(\mathbf{r}_{i j}\right)_{\mu}\left(\mathbf{r}_{i j}\right)_{\nu}}{L^{2}}\right]$ with $k(0)$ and $L \equiv 1$, where $r_{i j}=$ $\left|\mathbf{x}_{\mathbf{i}}-\mathbf{x}_{\mathbf{j}}\right|$.

Recently Yakhot [9] generelize the Polyakov's approach on the Burgers turbulence [8] for strong turbulence. He used the OPE approach to closed the equation for the velocity increment PDF and show that the PDF of longtudinal structure function $S_{q}=\left\langle(u(x+r)-u(x))^{q}\right\rangle=\left\langle U^{q}\right\rangle$, where $u(x)$ is the $x$-component of the three-dimensional velocity field and $r$ is the displacement in the direction of the $x$-axis satisfy the follwing equation for homogeneous and isotropic turbulence in the limit $r \rightarrow 0$ as,

$$
\frac{\partial}{\partial U} U \frac{\partial P}{\partial r}-B_{0} \frac{\partial P}{\partial r}=-\frac{A}{r} \frac{\partial}{\partial U} U P+\frac{u_{r m s}}{L} \frac{\partial^{2}}{\partial U^{2}} U P
$$

where $A=\frac{3+B}{3}, B=-B_{0}>0$ and for the NavierStokes turbulence it has been shown that $B \sim 20$ can be derived by a self-consistent calculations [9]. Last term in the right hand side is responsible for the breakdown of Galilien invariance in the limited Polyakov sense, which means that the single-point $u_{r m s}$ induced by random forcing enter the resulting expression for velocity increment PDF's.

Now one can show that the probablity density and as a result the conditional probablity density of velocity difference satisfy the Kramers-Moyal's evolution equation [11:

$$
-\frac{\partial P}{\partial r}=\sum_{n=1}^{\infty}(-1)^{n} \frac{\partial^{n}}{\partial U^{n}}\left(D^{(n)}(r, U) P\right)
$$

where $D^{(n)}(r, U)=\frac{\alpha_{n}}{r} U^{n}+\beta_{n} U^{n-1}$. We have found that the coefficients $\alpha_{n}$ and $\beta_{n}$ depend on $A$ and $B, u_{r m s}$ and inertial length scale $L$ which are given by $\alpha_{n}=(-1)^{n} \frac{A}{(B+1)(B+2)(B+3) \cdots(B+n)}$ and $\beta_{n}=$ $(-1)^{n} \frac{u_{r m s}}{L} \frac{1}{(B+2)(B+3) \ldots(B+n)}$ where $\beta_{1}=0$ by homogeneity [11]. The coefficients $D^{(n)}(r, U)$ are the small scale limit of the conditional moments [10]. They fully characterise the statsitics of eddy distribution in the inertial range which is defined as:

$$
\begin{gathered}
D^{(n)}\left(U_{2}\right)= \\
\lim _{r_{1} \rightarrow r_{2}} \frac{1}{r_{1}-r_{2}} \int\left(U_{1}-U_{2}\right)^{n} P\left(U_{1}, r_{1} \mid U_{2}, r_{2}\right) d U_{1}
\end{gathered}
$$

It is noted that $P\left(U_{1}, r_{1} \mid U_{2}, r_{2}\right)$ statisfies the equation (7). The Kramers-Moyal coefficients are the main observables of a Markov process from which all the terms in the Kramers-Moyal operator will be determined. It is a well known theorem (Pawula theorem) of Markov processes that whenever the fourth order Kramers-Moyal coefficient tends to zero all the the other terms with higher order derivatives tend to zero [10]. So the distinction between Markov processes which the Fokker-Planck description when just the first two terms in the evolution operator in scale are important and the Markov processes which all the terms should be preserved is related with the coefficients too. Thanks to the detailed analysis carried over experimental data [7], the functional form of the first four Kramers-Moyal coefficients has been obtained. It is been observed that the fourth order conditional moment tends to zero from which by invoking to Pawula's theorem the Fokker-Planck equation is canditated. In the mean time the present authors have shown that the Kramers-Moyal coefficients which are derived from the Yakhot modelling are extremely consistent with the experimental observations [11]. It is interesting that the functional forms of the different coefficients $\left(D^{n}(U, r)\right)$ are identically supporting the experimental observations [7]. The intermittency exponent of the structure functions can be derived from equation $(7), \zeta(p)=\frac{A p}{B+p}$. It is easy to see that the ratio of different $\mathrm{KM}$ coeficients are controlled by the $B$ parameter. As it is obvious when $B \rightarrow \infty$ K41 scaling is recovered and $B \rightarrow 0$ produces the extreme case of multiscaling related to Burgers intermittency [9]. The Kramers-Moyal's description of PDF 
deformation in scale has been supported by exact computation also for the compressible turbulence in high Mach number limit 12]. In that case the numerical values of the $A$ and $B$ terms is determined without any needs to numerical estimation. Reminding the original idea for suggesting the Markovian property of energy cascade in scale we take a step further and calculate the more general objects of the cascade,i.e. the unfused multiscale correlations. Assuming the Markovian nature of velocity increments in scale and the proposed form of evolution operator $L_{K M}(U, r)$, one in principle can calculate any correlation among velocity increments in different scales:

$$
\begin{gathered}
\mathcal{F}_{n}\left(x \mid r_{1}, r_{2}, \ldots, r_{n}\right)=\left\langle U\left(r_{1}\right) U\left(r_{2}\right) \ldots U\left(r_{n}\right)\right\rangle= \\
\int d U\left(r_{1}\right) \ldots d U\left(r_{n}\right) U\left(r_{1}\right) \ldots U\left(r_{n}\right) P\left(U_{1}, r_{1} ; U_{2}, r_{2} ; \ldots ; U_{n}, r_{n}\right)
\end{gathered}
$$

The joint probability $P\left(U_{1}, r_{1} ; U_{2}, r_{2} ; \ldots ; U_{n}, r_{n}\right)$ can be calculated by advantage of Markovian property in terms of conditional probabilities, i.e.,

$$
\begin{aligned}
& P\left(U_{1}, r_{1} ; U_{2}, r_{2} ; \ldots ; U_{n}, r_{n}\right)=P\left(U_{1}, r_{1} \mid U_{2}, r_{2}\right) \times \\
& \times P\left(U_{2}, r_{2} \mid U_{3}, r_{3}\right) \ldots P\left(U_{n-1}, r_{n-1} \mid U_{n}, r_{n}\right) P\left(U_{n}, r_{n}\right)
\end{aligned}
$$

The conditional PDF of velocity increments can be written as a scalar-ordered operator as $P\left(U_{1}, \lambda_{1} \mid U_{2}, \lambda_{2}\right)=$ $\mathcal{T}\left(e_{+}^{\left(\int_{\lambda_{2}}^{\lambda_{1}} d \lambda L_{K M}\left(U_{1}, \lambda\right)\right)}\right) \delta\left(U_{1}-U_{2}\right)$. So in the calculation of n-point multiscale correlation a series of conditional operators would emerge in the integrand of (8). When some of the coordinates coalesce the conditional operator tends to a dirac delta function. The reduction of the conditional probability between the coalescing coordinates simplify the calculations. The only remaining conditional operator will be the probability of observing the typical velocity $U_{1}$ increment between one subclass of fused points conditioned on observing the typical velocity increment $U_{2}$ in the other subclass of fused points. Explicitly we examine the behaviour of $\mathcal{F}_{p+q}\left(\lambda_{1}, \lambda_{2}\right)$ defined in 1 , where $\lambda_{1}=\ln (L / r)$ and $\lambda_{2}=\ln (L / R)$.

$$
\begin{gathered}
\mathcal{F}_{p+q}\left(\lambda_{1}, \lambda_{2}\right)=\left\langle U^{p}\left(\lambda_{1}\right) U^{q}\left(\lambda_{2}\right)\right\rangle=\int d U_{1} d U_{2} \delta\left(U_{1}-U_{2}\right) \times \\
\times P\left(U_{2}, \lambda_{2}\right)\left(e^{-\left(\lambda_{1}-\lambda_{2}\right) L_{K M}^{\dagger}\left(U_{1}\right)} U_{1}^{p}\right) U_{2}^{q}
\end{gathered}
$$

We restrict the calculations to the GI invariant apprtoximation so that neglecting the $O\left(u_{r m s} r / L\right)$ operators in $L_{K M}(U, \lambda)$. The crucial point in the above approximation is that in the GI regime the Kramers-Moyal's coefficients are scale independent so that all the scale dependence of conditional probabilities would reveal as a simple subtraction of the two logarithmic scales, i.e. $\lambda_{1}-\lambda_{2}$ in the exponent. Because $L_{K M}^{\dagger}\left(U_{1}\right) U_{1}^{p}=\zeta(p) U_{1}^{p}$, we will obtain the proposed form of the fusion rules in (2) with $\zeta(p)=\frac{A p}{(p+B)}$. Any other multiscale correlation function is also tractable under the same approximations.

The Fusion rules first introduced [2, 4 ] through invoking two Kolmogorov type assumptions. The first one is the assumption of a scale invariant form for all the correlation functions in the inertial range. The second is called the universality meaning that when some arbitrary set of velocity differences in the correlation functions are fixed in the scale $L$, their precise choice will affect the correlation functions just as an overall factor. In terms of conditional averages the second proposition means that

$$
\left\langle U\left(r_{1}\right)^{p} \mid U\left(r_{2}\right)^{q}\right\rangle=S_{p}\left(r_{1}\right) \Phi_{p, q}\left(r_{2}\right)
$$

where it is assumed also that scale of $r_{2}$ is in the order of integral scale while the $r_{1}$ is in the inertial range. The function $\Phi_{p, q}\left(r_{2}\right)$ isa homogenious function with a scaling exponent $\zeta_{n}-\zeta_{p}$, and is associated with the remaining $n-p$ indices of $\mathcal{F}$. Mathematically the above conditional correlation is easily verified

$$
\left\langle U\left(r_{1}\right)^{p} \mid U\left(r_{2}\right)^{q}\right\rangle=S_{p}\left(r_{1}\right) U_{2}^{p} / S_{p}\left(r_{2}\right)
$$

In Yakhot modelling scaling hypothesis has been invoked from the very beginning of the theory when the relevant $\mathrm{OPE}$ terms has been chosen for closing the equation governing over the generating function of longitudinal velocity increments. But we are showing that at least in the framework of Yakhot modelling the universality proposition is the result of the Markovianity of the evolution of velocity increments in scale. On the other hand the necessary proof of Markovian property has been verified through the special scalar-ordered form of the conditional probabilities. This itself has arisen from the general invariances and scaling constraint of the NavierStokes Equation. So the universality condition in the language of multiscale correlation functions has in it's heart the very robust scaling invariance under the infinite parameter scaling group [1]. We should emphasise that non-universal effects of the large scale motions can also manifest through the scale dependent terms in the Kramers-Moyal operator. Still the general form of Universality assumption would be the leading behaviour while the $O\left(u_{r m s} r / L\right)$ will be the sub-leading corrections inducing the large scale effects [?].

Within the experimentally verified approximation that neglects the third and higher order KM coefficients [11,7], one can write the equivalent diffusion process in scale which dynamically gives the relation between velocity increments at two different scales. In fact approximating the KM equation with a Fokker-Planck evolution kernel can be interpreted as if velocity increment $U$ is evoluted in "scale" $\lambda$ (logarithmic length scale) by the langevin equation [10], $\frac{\partial U}{\partial \lambda}=\tilde{D}^{(1)}(U, \lambda)+\sqrt{\tilde{D}^{(2)}(U, \lambda)} \eta(\lambda)$, where $\eta(\lambda)$ is a white noise and the diffusion term acts as a multiplicative noise. Giving address to the Ito's prescription 10] the multipoint correlation function can be written in the form of a path integral as

$$
\begin{gathered}
\mathcal{F}\left(\lambda_{1}, \lambda_{2}\right)=\int \mathcal{D} U U^{p}\left(\lambda_{1}\right) U^{q}\left(\lambda_{2}\right) e^{\int_{\lambda_{1}}^{\lambda_{2}}\left(\frac{\frac{\partial U}{\partial \lambda}-D^{1}(U, \lambda)}{\sqrt{D^{2}(U, \lambda)}}\right) d \lambda} \times \\
\times P\left(U_{2}, \lambda_{2}\right)
\end{gathered}
$$


By a simple application of Bayesian rule probability density in the outer scale $\lambda_{2}$ can also be written as a path integral entering the information of the integral scale PDF which in a good approximation can be regarded as a Gaussian distribution [9]. Building up all the terms in a descriptive way the joint probability $P\left(U_{1}, \lambda_{1} ; U_{2}, \lambda_{2}\right)$ is represented as a path integral over all possible paths between $U\left(\lambda_{1}\right)$ and $U\left(\lambda_{2}\right)$ transfering in an intermittent way all the information of the integral scale into the calculation. Without further attempt for calculating the multiscale correlation by path integral representation, we turn our attention to the Langevin dynamics instead. The resulting process is the well known Kubo [10 oscillator multiplicative process. By using the Ito [10] prescription one can deduce that

$$
\delta_{\lambda_{1}} U(x)=\mathcal{W}\left(\lambda_{1}, \lambda_{2}\right) \delta_{\lambda_{2}} U(x)
$$

The multiplier $\mathcal{W}\left(\lambda_{1}, \lambda_{2}\right)$ can be easily derived in terms of $\alpha_{1}$ and $\alpha_{2}$ and Wiener process at two logarithmic scales as:

$$
\mathcal{W}\left(\lambda_{1}, \lambda_{2}\right)=e^{\left(-\alpha_{1}\left(\lambda_{1}-\lambda_{2}\right)+\sqrt{\alpha_{2}}\left[W\left(\lambda_{1}\right)-W\left(\lambda_{2}\right)\right]\right)} .
$$

Equation (12) encodes a simple cascade process. Cascade processes are simple and well known useful tools to describe the leading phenomenology of the intermittent energy transfer in the inertial range. Both anomalous scaling exponents and viscous effects [1] can be Under the framework of the derived KM equation we have shown that the equivalent cascade model from NavierStokes equation is derived by cutting the KM evolution operator after third term. In fact we have given a recipe for finding the equivalent approximate cascade model from Navier-Stokes equation. Structure functions are described in terms of multiplier $W\left(\lambda_{1}, \lambda_{2}\right)$ through $S_{p}(r)=C_{p}\left\langle[\mathcal{W}(r / L)]^{p}\right\rangle$ where from the Langevin equation pure power law arise in the high Reynolds regime $\left\langle[\mathcal{W}(r / L)]^{p}\right\rangle \sim(r / L)^{\zeta(p)}$. In this approximation the scaling exponents would be $\zeta(p)=-p \alpha_{1}+p(p-1) \alpha_{2} / 2$. From the direct calculation of Langevin equation one can easily find the behavior of the multiscale correlation function $\mathcal{F}_{p+q}(r, R)$. In the same framework, it is staightforward to show that

$$
\begin{array}{r}
\mathcal{F}_{p+q}(r, R) \sim\left\langle[\mathcal{W}(r, R)]^{p}[\mathcal{W}(R, L)]^{q}\right\rangle \\
\sim\left\langle\left[\mathcal{W}\left(\frac{r}{R}\right)\right]^{p}\right\rangle\left\langle\left[\mathcal{W}\left(\frac{R}{L}\right)\right]^{q}\right\rangle \sim S_{p}(r) S_{p+q}(R) / S_{p}(R)
\end{array}
$$

The independence of multipliers in two diferent scales is allways assumed for the underlying cascade process otherwise the following relation would not be held. Our modelling equivalently encodes the following requirement by the obvious independency of increments in a Wiener process. Recently Benzi and coworkers [6] analysed multiscale correlation functions from high Reynolds experiments and synthetic signals. They have elegantly seek whether the fusion rules (3) are compatible with the random cascade phenomenology. Their main result is that all multiscale correlation functions are well reproduced in their leading term $\frac{r}{R} \rightarrow 0$ by a simple uncorrelated random cascade. In Yakhot modelling of the dynamics of the longitudinal velocity increments in scale all the above results are recovered in the framework of Fokker-Planck approximation. It is also interesting also to seek the limiting behaviour of the Multiscaling correlation function for Burgers turbulence which is tractable by taking the limit of $B \rightarrow 0$ in our formulation. Equation (3) shows that multiscaling correlation function will be independent of the outer scale $R$ which is consistent with our knowledge about Burgers turbulence 13]. We think that preserving all the terms in KM equation would give the full information of cascade in length scale and this would give an answer to the question that wether there are other subleading processes acting for energy transfer from large to small scales. Preserving the GI breaking terms in the corresponding stochastic processes would also give the important unanswered question regarding the effect of uneven PDF's of velocity increments on the cascade models.

We thank Uriel Frisch, Itammar Procaccia and Victor Yakhot for useful remarks and comments. M.R. Rahimi Tabar wish to acknowledge Observatoire de la Côte d'Azur where some part of this work is done and U. Frisch for the kind hospitality.

[1] U. Frisch " Turbulence" Cambridge University Press (1995)

[2] V.S.L'vov and I. Procaccia, Phys.Rev.Lett. 76, 2898 (1996).

[3] V.S.L'vov and I. Procaccia, Phys.Rev. E 54, 6268 (1996).

[4] G. Eyink, Phys.Lett. A 172, 355 (1993); G. Eyink, Phys.Rev. A 48, 1823 (1993).

[5] A.L. Fairhall, B. Druhva, V.S. L'vov, I. Procaccia, and K.S. Sreenivasan( to be published).

[6] R. Benzi, L. Biferale, and F. Toschi, Phys.Rev.Lett. 80, 3244 (1998).

[7] R. Friedrich, J. Peinke, Phys. Rev. Lett. 78,863 (1997) ;B.Chabaud, A. Naert, J. Peinke, F. Chilla, B. Castaing and B. Hebral, Phys. Rev. Lett. 73, 3227 (1994).

[8] A. Polyakov, Phys. Rev. E 52, 6183 (1995)

[9] V. Yakhot, Phys. Rev. E, 57, 1737 (1998)

[10] N. G. van Kampen,"Stochastic Prosses in Physics and Chemistry(Elsevier, Amesterdam 1990); H. Risken," The Fokker-Planch Equation" (Springer-Verlag, 1984)

[11] Jahanshah Davoudi and M. R. Rahimi Tabar," Phys.Rev.Lett. 82, 1680 (1999).

[12] M.R.Rahimi Tabar, Jahanshah Davoudi, A. Rastegar, IPM Preprint.

[13] U. Frisch, private communication. 